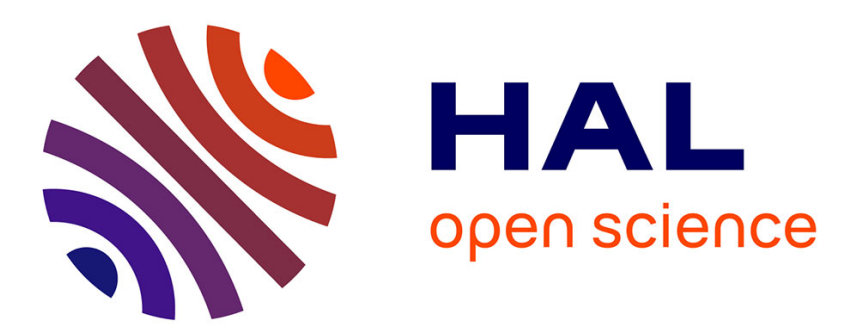

\title{
Comparison between empirical or functional sinks of organs - Application on Tomato plant
}

Baogui Zhang, Meng Zhen Kang, Véronique Letort, Xiujuan Wang, P. de Reffye

\section{- To cite this version:}

Baogui Zhang, Meng Zhen Kang, Véronique Letort, Xiujuan Wang, P. de Reffye. Comparison between empirical or functional sinks of organs - Application on Tomato plant. 2009 Plant Growth Modeling And Applications, Nov 2009, Beijing, China. pp.191-197. hal-00546803

\section{HAL Id: hal-00546803 https://hal.science/hal-00546803}

Submitted on 15 Dec 2010

HAL is a multi-disciplinary open access archive for the deposit and dissemination of scientific research documents, whether they are published or not. The documents may come from teaching and research institutions in France or abroad, or from public or private research centers.
L'archive ouverte pluridisciplinaire HAL, est destinée au dépôt et à la diffusion de documents scientifiques de niveau recherche, publiés ou non, émanant des établissements d'enseignement et de recherche français ou étrangers, des laboratoires publics ou privés. 


\title{
Comparison between empirical or functional sinks of organs - Application on Tomato plant
}

\author{
Baogui Zhang*, Mengzhen Kang ${ }^{\dagger}$, Véronique Letor ${ }^{\ddagger}$, Xiujuan Wang*, Philippe de Reffye ${ }^{\S}$ \\ * Key Laboratory of Plant-Soil Interactions, Ministry of Education, College of Resources \\ and Environment, China Agricultural University, Beijing 10013, China \\ $\dagger$ LIAMA \& NLPR, Institute of Automation, Chinese Academy of Sciences, Beijing, 100080, China \\ ${ }^{\ddagger}$ Ecole Centrale Paris, Laboratory of Applied mathematics, 92290 Chatenay-Malabry, France \\ ${ }^{\S}$ Cirad-amis, TA 40/01 Ave Agropolis, 34398 Montpellier cedex 5, France \\ IINRIA-Futurs, 4, rue J. Monod,91893 Saclay cedex, France \\ *Email: baogui.zhang@gmail.coms
}

\begin{abstract}
Biomass partitioning among organs depends on their sink strengths, i.e. their capacity to attract assimilates. Using a descriptive approach, where plant development is driven by thermal time, and empirical laws fitted from experimental data, it is possible to trace back by inverse method the dynamics of biomass partitioning among organs. However, the descriptive sink function suffers from the drawbacks that organ development and biomass accumulation are not interactively related. Moreover, many parameters are required and are difficult to be measured accurately. In this paper an alternative organ sink strength definition is proposed, in which the organ sink size is related to the maximum organ size, which in turn depends on its primordium size. The sink strength increases proportionally to its size at the early growth stage and decreases by dampening when it approaches the final size. Comparisons to the standard empirical sink function used in the GreenLab model were conducted on tomato plants. The new functional sinks are more biologically relevant and simulated rather adequately the organ biomass evolution. Further improvement is ongoing to increase simulation accuracy.
\end{abstract}

\section{Introduction}

Biomass partitioning among organs depends on their sink strength. This notion emerged in the 1970s, defines the capacity of a growing zone to get carbohydrates when competing with other growing zones of a plant [1]. Expressions of this notion in models have many variants: sink mass, number of active cells, sink activity, flow mass, potential growth rate under non limiting conditions [2], and some other involves divers processes: unloading from the sieve elements, metabolism in the cell wall, uptake, conversion and storage of dry matter. These definitions suffer from the drawbacks that sink strength does not have intrinsic nature, its value depends on either source strength or environmental conditions; moreover, it is difficult to quantify experimentally.

To overcome the difficulties associated to experimental measurements of sink activity, models such as GreenLab [3], [4] provide an alternative approach. By fitting the model on experimental data, it extracts the sink dynamics of individual organs. In this context, Greenlab can be seen as a source-sink solver. It offers new insights in the plant internal assimilate competition. In the previous versions of the GreenLab model, sink strength was described with generic shapes of beta law density functions. This allowed extracting the sink dynamics of individual organs during their growth. The model has been used to simulate Arabidopsis thaliana [5], maize [4], beech tree (Fagus sylvatica) [6], tomato [7], Chinese pine [8], this modeling approach was purely mathematical, descriptive and empirical, but not explicative. However it is possible to make a compromise between a mathematical expression of a function that is noexplicative but flexible enough to reproduce any variation of sink strength with time and a definition that is biologically based. In this paper, we propose a new definition of organ sink strength that would relate more closely to the biological processes.

Tomato is an important horticultural plant. Its production and market value depend not only on the quantity of fruits but also on their size. When fitting the organ mass of tomatoes to get the parameters of sink strength with the usual beta law density functions, great discrepancies occurred between simulated and observed organ sizes on the phytomers bearing the truss. This can be physiologically explained by the fact that tomatoes have an indeterminate growth habit, after flowering, the vegetative and reproductive growths coexist. Therefore the phytomers bearing the trusses have different assimilate partitioning rules than the 
others. It was first attempted to add new parameters to the model to simulate these two types of phytomers alternating periodically along the main stem. In a second step, a new modelling approach was introduced to propose an expression of sink strength that would be more biologically relevant and that would account for the allocation changes observed in tomato phytomers. In this paper we compare the classical definition of sink strength used in the GreenLab model to the two new methods based on a case study on tomato.

\section{Materials and Methods}

\subsection{Description of the GreenLab model}

GREENLAB is a functional-structural model that simulates plant development, growth and their retroactions to generate plant plasticity at organ level [3], [4]. The time step of the model is a growth cycle (GC), corresponding to a phyllochron for crops (thermal time between appearances of two successive leaves on the main stem). One component of the model is the simulation of plant organogenesis at each GC. Another component, the functional part, describes the expansion of individual organs as the result of biomass production and repartition at each GC. The organogenesis model is relatively simple for crops compared to trees. In the following section we focus on the functional part where the new sink model is introduced.

There are several assumptions linked to biomass partitioning in GreenLab: (1) organs directly access to a common pool of assimilate, ignoring the transport resistance dependent on source distance; (2) assimilate is converted directly to biomass without any metabolic cost, thus in the paper, biomass and assimilate are used interchangeably; (3) biomass allocated to an individual organ depends on the relative sink strength of the plant organs and on the source-to-sink ratio (Q/D: Quantity of biomass production vs. Demand) at each GC.

2.1.1. Biomass production. Assimilate production from photosynthesis is computed using Beer-Law approach, similar to process based models:

$$
Q(n)=E(n) \frac{S_{p}}{r}\left(1-\exp \left(-k \frac{S_{L}}{S_{p}}\right)\right)
$$

where $E(n)$ is a parameter integrating environmental conditions at nth GC that influence on the resource supply for plant growth; $r$ is a parameter that is inversely proportional to the efficiency of resource acquisition. $Q(n)$ is the fresh biomass produced at $n$th GC, $k$ is analogous to the extinction coefficient of Beer-Lambert's Law; it is set to 0.8 for tomato here. $S_{L}$ is the active leaf area of the plant; $S_{P}$ is the theoretical projection surface of an individual plant. Their ratio represents a leaf area index for an individual plant. In practice, $S_{P}$ is estimated as a root of equation (1).
2.1.2. Biomass repartition and organ sink function. At each GC $n$, the amount of assimilate $Q(n)$ is allocated to expanding organs proportionally to their current relative sink strength. Let $f_{o}(j)$ be the sink strength of an organ that appeared at GC $j$. The total demand of plant $D(n)$ of all expanding organs at $n$th GC:

$$
D(n)=\sum_{o} \sum_{j=n-t_{o}+1}^{n} N_{o}(j, n) f(j)
$$

where $N o(j, n)$ is the number of organs of type $o$ that appeared at cycle $j$ when the plant is $n$ GC aged. For tomato plants, organ type $o$ includes leaf blade $(b)$, petiole $(p)$, internode $(i)$ fruit $(f)$ and $\operatorname{root}(r)$ in the aerial part. For an organ created at GC $j$, at a given plant age $n(n>j)$, the biomass increment of an organ is proportional to its sink function and ratio between quantity of biomass producted in the previous cycle and the plant demand at current cycle, which is an indicator of plant competition:

$$
\triangle q_{o}(j, n)=f(j) \frac{Q(n-1)}{D(n)}
$$

The organ biomass is the result of biomass accumulation since its initiation is:

$$
q_{o}(j, n)=\sum_{i=j}^{n} \triangle q_{o}(j, n-j+1)
$$

The organ sink functions $f o(j)$ is the kernel of biomass allocation and organ growth. We present three different ways to define these functions, including both empirical and functional approaches.

\section{(1) Classical Beta law (Beta 1)}

In this case, the flux of biomass entering into an organ during growth is controlled by a fixed empirical function whose parameters are supposed to be stable for a given type of organ. Each organ has an expansion duration $t_{o}$ which can vary according to the organ position in the plant architecture. The relative sink strength for each type of organ was defined as a function of its age expressed in GC:

$$
f_{o}(i)=P_{o} F_{o}(i)
$$

In this equation, $P_{o}$ is the coefficient of sink strength associated with organs type $o$. For leaf blade, $P_{b}=1$ is set as a reference. $F_{o}(i)$ is an organ type-specific function of sink variation during a duration $t_{O}$ expressed in GC, described with Beta function as follows:

$$
F_{o}(i)= \begin{cases}g_{o}(i) / \mu & 1 \leq i \leq t_{o} \\ 0 & i>t_{o}\end{cases}
$$

where

$$
\begin{array}{r}
g_{o}(i)=(i-0.5)^{a-1}\left(t_{o}-i+0.5\right)^{b-1} \\
\mu_{o}=\max \left\{g_{o}(i) \mid 1 \leq i \leq t_{o}\right\}
\end{array}
$$


The parameters $a_{o}$ and $b_{o}$ vary with organ type. This function is flexible to describe the sink variation and can be fitted to data by optimization.

(2) Beta law with two kinds of phytomers (Beta 2)

This method was designed for the particular case of tomato plants. In this case, Beta laws are still used for organ sink functions, but the phytomers bearing the truss are explicitly distinguished from the others. Parameter $a \_b$ and $a \_i$ are respectively the scale factors for the sink of leaves (blades and petioles) and internodes of the phytomers with truss compared to the phytomers without truss.

\section{(3) Functional sink function (New sink)}

Here we propose a new sink strength function, where the potential organ size is dependent on $Q / D$. Two phases are considered: organ creation (linked to cell division) and organ expansion (linked to cell expansion). An organ gets its initial biomass at creation proportionally to the sourceto-sink $(Q / D)$ ratio at its creation cycle:

$$
q_{o}(j, j)=C_{o} \frac{Q(j-1)}{D(j)}
$$

For simplicity, in the present version, only $Q / D$ of the first cycle of the organ creation was considered, although it takes several growth cycles for an organ formation. It is assumed that there is a maximum size of organ, which is proportional to its initial biomass according to the equation:

$$
q_{\max , o}(j)=X_{o} q_{o}(j, j)
$$

$X_{O}$ is a parameter corresponding to the expansion coefficient of cells of the organ. In computing the organ maximum size as described above, it is in fact implicitly assumed that the cell number is proportional to the initial biomass of organ, and $X_{o}$ is related to the average cell size of organ type o. The expansion of an existing organ is proportional to its current biomass and the growth rate is limited by the maximum sink size:

$$
f_{o}(j, n)=C_{o}\left(\frac{q_{o}(j, n-1)}{q_{\max , o}(j)}\right)\left(1-\frac{q_{o}(j, n-1)}{q_{\max , o}(j)}\right)
$$

Thus, differing from the empirical function shown in Eqn. 5 and Eqn. 6, the new sink strength of organ depends not only on its own age, but also on the plant age and its current size. The new parameters to be identified are $C_{o}$ and $X_{o}$.

Based on these dynamical equations, the plant growth can be simulated and the organ size computed depending on biomass production and on the number and strength of sinks that share the biomass at a given time. Parameter optimization of the model uses the generalized least square method described by Zhan et al. [9] and Guo et al. [4]. From the measurements taken on the plant architecture, it is possible with the GreenLab model to compute the source and sink parameters, and thus to get the evolution of both growth and demand of the plant during the duration of its development. A 3-D simulation of the tomato plant was realized with the GreenSciLab software developed by our group [10].

\subsection{Plant growth sampling and measurement}

Tomato (Solanum lycopersicum L.; previously named Lycopersicon esculentum Mill.) cultivar Hybrid-CN 9 were grown in a greenhouse of CASA, Beijing $\left(39.59^{\circ} \mathrm{N}\right.$, $116.17^{\circ} \mathrm{E}$ ), from March to June 2006. Seeds were germinated in peat compost. Plants with 3 to 4 true leaves were transplanted into soil mixed with manure and a 15-15-15 N-P-K fertilizer. All branches were removed as soon as they appeared. Flowers were pollinated by released bees. Air temperature inside the greenhouse varying from 15 to 30 degrees was recorded once an hour. Sixteen plants were sampled at four dates (18 April, 8, 23 May and 7 June), from the start of flowering (with 8 leaves on the main stem) to the appearance of the 6 th truss (with 26 to 27 leaves). Four relatively uniform-sized plants were taken at each sample date. Plants were separated into root system and shoot with the later further divided into phytomers. For each phytomer, length, diameter and fresh and oven-dry weight $\left(75^{\circ} \mathrm{C}\right.$ to constant weight) of the internode, fresh and dry weight leaf blade, petiole, fruits of the whole truss (if present) were determined.

\subsection{Statistical analysis}

For model evaluation, simulated biomass of organs (blade, petiole, internode and fruits) were compared with the corresponding observed values. The statistics used to evaluate model performance were the total normalized root mean square error (TNRMSE), where $s$ and $x$ are respectively the simulated and observed organ biomass on the $i$ th phytomer, and $n$ is the number of pairs of simulated and observed data, $o$ represents the four types of organs namely blade $(b)$, petiole $(p)$, internode $(i)$ and fruits $(f)$. TNRMSE is calculated as following:

$$
T N R M S E=\sum_{o}^{b, p, i, f} \frac{\sqrt{\frac{\sum\left(s_{i}-x_{i}\right)^{2}}{n}}}{\frac{\sum\left(x_{i}-\bar{x}\right)^{2}}{n-1}}
$$

The smaller the TNRMSE, the better the simulation.

\section{Results and discussions}

\subsection{Organ biomass and simulation with the three sink functions}

As fresh weight of fruits is of interest in tomato, analysis of biomass production and repartition among organs was 
performed mainly in terms of fresh weight (Fig. 1), and dry matter analysis gave similar results (Fig. 2). Because of space limitation, only two of the four plants (one with fresh weight and another with dry weight) at the last harvest date was presented, all the 16 sampled plants at 4 harvest dates were analyzed, and gave similar results (data not shown). Biomass profiles of the leaves and internodes along the stem are bell-shaped with certain fluctuations (Fig. 1 A, B, C). From the stem base to top, the biomass of the phytomers increases, reaching a maximum at the phytomer 10 or 11 which bear the second inflorescence (Fig. $1 \mathrm{D}$ ) and decreased gradually. The organ biomass of the last 6 phytomeres located at the stem top are still expanding and have not yet their final size. Similar organ size profiles have been observed in maize [4], and cucumber [11]. The pattern of organ biomass distribution along the stem is related to the source-to-sink ratio of the plant when the organs are initiated [11]. In fact, at early stages of plant development, the organ growth depends on the seed reserve and assimilates from some small leaves. As the plant grows, the leaf surface area increases, thus the assimilate supply and source-to-sink ratio increase until the appearance of the fruits, particularly the initiation the second truss.

For sink function Beta-1 with the descriptive (developmental) i.e. empirical approach, biomass accumulation of an organ is determined by sink size (strength), duration of expansion, sink variation function and source-to-sink ratio of the plant during the expansion period. Sink size and variation are same for all the organs of a given category regardless of neither their position on the stem nor the time of their creation. Thus the evolution of organs biomass along stem follows that of the source-to-sink ratio (data not shown). As the latter is calculated at the whole plant level, local fluctuations are suppressed, resulting thus in a smooth organ biomass profile (dashed lines in Fig. 1). In the functional sink approach (New sink), the organ maximum size, and thus its sink size, depends on the plant source-to-sink ratio at the organ creation. So the organs of the same type may have different sink size according to when, and implicitly where, the organ is created. Hence the functional sink can simulate the fluctuation of organ size along the stem (Fig. 1).

The biomass of the leaves on the phytomers bearing the truss are smaller than the values predicted by the Beta- 1 model, while those of the internodes are greater than the simulation (underestimated by the model), giving a zigzag aspect (Fig. $1 A, B, C$ ). In the tomato cultivar, shoot apex is converted into an inflorescence (develop to truss) after 8 (less frequently 7 to 9 ) leaves have formed and a new vegetative shoot arises from a meristem at the axil of the youngest leaf. The new meristem produces a sympodial segment composed of three phytomers before again converting into an inflorescence, and the above described process reiterates. So the main stem of tomato is composed of two types
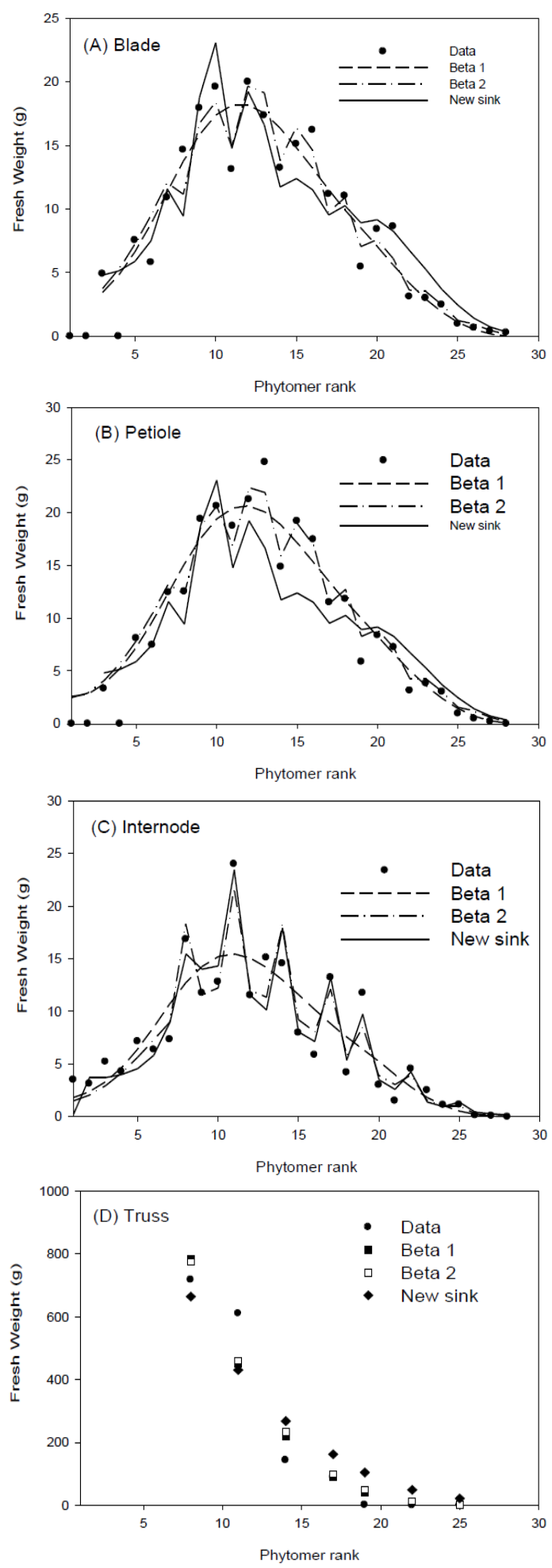

Figure 1. Fitting of organ fresh biomass according to phytomer rank with empirical (Beta1 and Beta2) and functional sink strength functions (New sink). A: leaf blade; $\mathrm{B}$ : petiole; $\mathrm{C}$ : internode and $\mathrm{D}$ : total fruits of the truss. Only one of the four plants analyzed is presented here because of space limitation. 


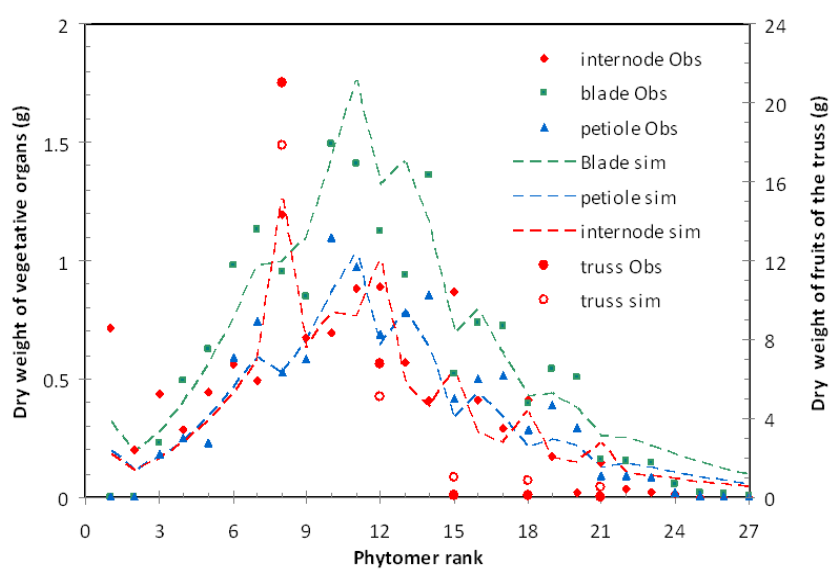

Figure 2. Fitting of organ dry weight with functional sink strength function (new sink). Because of page limitation, only one of the four plants harvested and analyzed for the last harvest date is presented here, note that the plant had different topological structure than that of another plant shown in the Fig. 1.

of segments: one is the primary segment formed before flowering and the other is the sympodial segment [12].

For the descriptive sink function Beta-2 and the

Table 1. Comparison of model parameters and simulation error

\begin{tabular}{|c|c|c|c|}
\hline Parameters & Beta-1 & Beta- $2 \dagger$ & New sink $\ddagger$ \\
\hline$P_{b}$ & 1 & 1 & - \\
\hline$P_{p}$ & 1.09 & 1.12 & - \\
\hline$P_{i}$ & 0.93 & 0.69 & - \\
\hline$P_{f}$ & 61.3 & 53.7 & - \\
\hline$C_{b}$ & - & - & 0.087 \\
\hline$C_{p}$ & - & - & 0.087 \\
\hline$C_{i}$ & - & - & 0.045 \\
\hline$C_{f}$ & - & - & 2.58 \\
\hline$b_{b}$ & 2.62 & 1.85 & - \\
\hline$b_{p}$ & 2.45 & 1.76 & - \\
\hline$b_{i}$ & 3.32 & 2.60 & - \\
\hline$b_{f}$ & 3.57 & 3.11 & - \\
\hline$a \_b$ & - & 0.76 & 1.00 \\
\hline$a_{-} i$ & - & 1.75 & 2.10 \\
\hline$X_{b}$ & - & - & 14.1 \\
\hline$X_{p}$ & - & - & 17.3 \\
\hline$X_{i}$ & - & - & 21.8 \\
\hline$X_{f}$ & - & - & 41.6 \\
\hline TNRME & 11.6 & 5.09 & 8.87 \\
\hline
\end{tabular}

$P_{o}(o=$ blade, petiole, internode, fruits):

relative sink size of organ with that of blade set to 1 ;

$C_{o}$ ( $o=$ blade, petiole, internode, fruits): sink size of organs ;

$X_{O}$ ( $o=$ blade, petiole, internode, fruits): expansion coefficient;

$\dagger a \_b$ and $a \_i$ are scale factors for the sink of phytomers

with truss compare to the phytomer without truss;

$\ddagger T N R M E$ : total normalized root mean error $i$ calculated with 10 .

functional sink approach named 'NewSink', two types of phytomers we distinguished the phytomers bearing the truss (inflorescences) from the others. Both models simulated

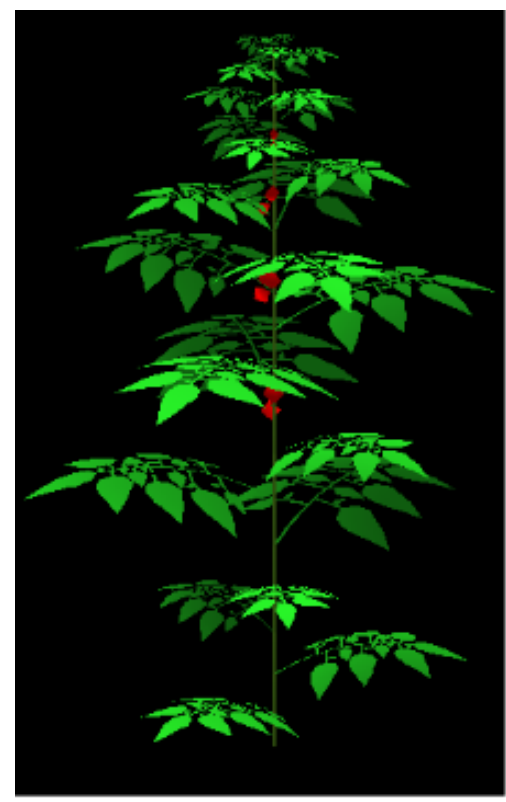

Figure 3. Three-D visualization of a tomato plant at 27 growth cycles

rather faithfully the 'Zigzag' phenomenon. The leaves of the first two (particularly the first) truss-phytomers were underestimated by the third model 'NewSink' (Fig. $1 A$ and Fig. $1 B$ ). In conformity with the concept of common assimilate pool, the organs growing simultaneously competes for assimilates. In the present study, all the fruits on the truss are pooled together to give a 'metafruit' with large biomass. It induces a great sink size for fruits which may explain the underestimation of leaves, internodes and the overestimation of the truss-bearing phytomers mass. In the ongoing work, target data will include weights of individual tomato fruits instead of that of the truss. Hopefully this would improve the simulation accuracy of the new sink model.

The new sink function gives satisfactory results with a total normalized root mean error being intermediate of simulation between that using the descriptive function Beta 1 and Beta 2 (Table 1). and the 3-D visualization of a tomato plant looks rather realistic (Fig. 3). It is interesting to note that parameters of sink strength of leaf blade and petiole are the same regardless of the models. We recall that allometries $a \_b$ and $a \_i$ are scale factors for the sink of phytomers with truss compare to the phytomer without truss. For the functional model (NewSink), the scale factor $a \_b$ for leaves is 1 , suggesting that there was no difference between the leaves on the truss-bearing phytomers and these on other phytomers. On the contrary, in the Beta-2 model, the factor is less than 1 , which suggests that the leaves on the phytomer bearing the inflorescence (or truss) have intrinsic smaller sink size. The real value is probably that obtained with the model Beta- 2 as it fits best the observation data 
either visually (Fig. $1 \mathrm{~A}, \mathrm{~B}, \mathrm{C}$ ) or in term of the simulation error (Table 1). As shown in formula (8), $X_{o}$ and $C_{o}$ are multiplicative factors: they are both proportional (also not linearly) to the organ biomass accumulation, thus the ratio

$$
\frac{X_{f} C_{f}}{X_{b} C_{b}}
$$

represents to a certain extend the relative sink strength of fruits compared to that of blades, which were interesting not different among the three models (table 1).

In brief, these three methods give comparable results when tested on tomato data. But the functional sink approach (NewSink), presents the advantage that modeling and simulating the biomass partitioning and accumulation in the organ require less parameters than the two others. In particular, the expansion time of organ which is difficult to measure accurately is no longer needed.

\subsection{Biological relevance of the descriptive and functional approaches}

In the descriptive sink model (Beta1 and Beta2), the duration of expansion of an organ is fixed: both the start and end dates of the expansion are determined by thermal time. Once the expansion time expires, the organ growth stops, even if the biomass accumulation is very small due to a weak source-to-sink ratio in the plant during this period. In this sense, the development and the biomass accumulation of the organ are uncoupled.

On the contrary, with the functional approach, an organ stops to accumulate biomass only when it reaches the volume defined by its maximum sink size. As observed by many researchers, late developed tomato fruits stay for many weeks in a growth latency caused by the competition for assimilates among fruits and they can finally resume their growth as soon as the previously growing fruits ripe [11], [13].

Furthermore, in the functional sink function, the biological meaning of the model parameters is more evident. The biological process of organ growth and most recent advances in plant developmental research are taken into account. For example, the formula (7) represents the fact that the initial biomass of an organ (primordium) depends on source-tosink ratio of the plant at that time. This is in conformity with the finding of numbers of researchers. Liu et al. [14] and Baldet et al. [15], [16] demonstrated that increase in assimilate availability increased the cell proliferative at very early stages of flower development and thus the final fruit size in tomato. Similarly, Mizukami and Fischer showed that altering the function of the AINTEGUMENTA (ANT) gene in Arabidopsis modified the final size of petal and leaves by influencing cell proliferation during organogenesis [17]. It means that the total number of cells and ultimately their expansion, determine intrinsic organ size. It should be noticed that for the organs of a given type, there is no common maximum size but various maximum sizes corresponding to their individual primodium sizes.

\section{Conclusion}

Until now the empirical function of GreenLab has always fulfilled the role of source and sink solver for the various crops studied. But the functional sink approach allows more flexible adequate simulation of the organ biomass accumulation; it requires less model parameters and is more closely related to the underlying biological process. Improvement of this model is ongoing: sink size will be considered in terms of individual tomato fruit instead of the truss. The new formulation will be tested on more species in diverse situations.

\section{Acknowledgements}

This study was supported by Chinese Ministry of Science and Technology in the project of National High Technology of Research and Development of China (grant No $2006 A A 10 A 303-1)$, and the Natural Science Foundation of China (grant No 60703043).

\section{References}

[1] J. Farrar, "Sink strength: What is it and how do we measure it? a summary," Plant, Cell and Environment, vol. 16(9), pp. 1045-1046, 1999.

[2] E. Heuvelink, "Re-interpretation of an experiment on the role of assimilate transport-resistance in partitioning in tomato," Annuals of Botany, vol. 78, pp. 467-470, 2004.

[3] P. de Reffye, E. Heuvelink, Y. Guo, B. Hu, and B. Zhang, "Coupling process-based models and plant architectural models: A key issue for simulating crop production," in Crop modeling and decision support, in: cao, w.x. and white, j.w. and wang, e.l. ed. Springer/Tsinghua Univesity Press, 2009, pp. 130-147.

[4] Y. Guo, Y. Ma, Z. Zhan, B. Li, M. Dingkuhn, D. Luquet, and P. de Reffye, "Parameter optimization and field validation of the functional-structural model greenlab for maize," Ann BotLondon, vol. 97, pp. 217-230, 2006.

[5] A. Christophe, V. Letort, I. Hummel, P. Courn “.'de, P. De Reffye, and J. Lecoeur, "A model-based analysis of the dynamics of carbon balance at the whole-plant level in arabidopsis thaliana," Functional Plant Biology, vol. 35, pp. 1147-1162, 2008

[6] V. Letort, P. Cournede, A. Mathieu, P. De Reffye, and T. Constant, "Parametric identification of a functional-structural tree growth model and application to beech trees (Fagus sylvatica)," Funct Plant Biol, vol. 35, pp. 951-963, 2008. 
[7] Q. Dong, G. Louarn, Y. Wang, J. Barczi, and P. de Reffye, "Does the structure-function model greenlab deal with crop phenotypic plasticity induced by plant spacing? a case study on tomato," Ann Bot-London, vol. 101, pp. 1195-1206, 2008.

[8] H. Guo, V. Letort, L. Hong, T. Fourcaud, P. Cournede, Y. Lu, and P. de Reffye, "Adaptation of the greenlab model for analyzing sink-source relationships in chinese pine saplings," in Second International Symposium on Plant Growth Modeling, Simulation, Visualization and their Applications " C PMA; ${ }^{-06}$, in: fourcaud t, zhang xp. eds. ed., ser. Crop modeling and decision support. IEEE Computer Society, Beijing, China, 2006, pp. 236-243.

[9] Z. Zhan, P. de Reffye, F. Houllier, and B. Hu, "Fitting a structural-functional model with plant architectural data," PMA'03, Beijing, China, Tsinghua University Press and Springer, 2003.

[10] M. Kang, J. Evers, J. Vos, and P. de Reffye, "The derivation of sink functions of wheat organs using the greenlab model," Annals of Botany, vol. 101, pp. 1099-1108, 2008.

[11] A. Mathieu, B. Zhang, E. Heuvelink, S. Liu, P. Courn '.'de, and P. de Reffye, "Calibration of fruit cyclic patterns in cucumber plants as a function of source-sink ratio with the greenlab model," in Proceedings of the 5th International Workshop on Functional Structural Plant Models, j.lovett doust and 1. lovett doust ed. Napier, New Zealand., November 2007, no. 5, pp. $1-4$.

[12] J. Atherton and G. Harris, Flowering, in: j.g. atherton, and j. rudich ed. Chapman and Hall, London, 1986.

[13] N. Bertin, "Analysis of the tomato fruit growth response to temperature and plant fruit load in relation to cell division, cell expansion and dna endoreduplication," Annals of Botany, vol. 95, pp. 439-447, 2005.

[14] J. Liu, B. Cong, and S. Tanksley, "Generation and analysis of an artificial gene dosage series in tomato to study the mechanisms by which the cloned quantitative trait locus fw 2.2 controls fruit size," Plant Physiology, vol. 132, pp. 292-299, 2003.

[15] P. Baldet, C. Devaux, C. Chevalier, and et al., "Contrasted responses to carbohydrate limitation in tomato fruit at two stages of development," Plant, Cell and Environment, vol. 25, pp. 1639-1649, 2002.

[16] P. Baldet, M. Hernould, F. Laporte, C. Chevalier, and et al., "The expression of cell proliferation - related genes in early developing flowers is affected by a fruit load reduction in tomato plants," Journal of Experimental Botany, vol. 57, pp. 961-970, 2006.

[17] Y. Mizukami and L. Fischer, "Plant organ size control AINTEGUMENTA regulates growth and cell numbers during organogenesis," Proc Natl Acad Sci, vol. 97, pp. 942-947, 2000 . 